\title{
Ocorrência de ovos de helmintos em hortaliças comercializadas na cidade de Apucarana (PR)
}

\section{Occurrence of helminth eggs on vegetables sold in the city of Apucarana (PR)}

\author{
Angélica Albuquerque Tomilhero Frias ${ }^{1}$; Joseane Balan da Silva²; Heloísa de \\ Camargo Tozato ${ }^{3}$
}

\section{Resumo}

As doenças veiculadas por hortaliças representam um importante problema de saúde pública, pois estima-se que milhões de pessoas de todo o mundo estejam acometidas por parasitoses. O presente estudo teve por objetivo quantificar e identificar o índice de contaminação por ovos de helmintos em hortaliças do tipo agrião e almeirão comercializadas em quatro grandes supermercados da cidade de Apucarana (PR). Foram analisadas 128 amostras de hortaliças sendo 64 de almeirão e 64 de agrião. As hortaliças foram desfolhadas, pesadas e lavadas em solução lauril sulfato triptose a $1 \%$. Observouse um índice elevado de contaminação nas duas variedades estudadas, com maior prevalência no almeirão. Foram encontrados ovos de Ancylostoma sp., Ascaris sp., Diphilobotrium sp., Dipylidium sp., Hymenolepis sp., Taenia sp. e Enterobius sp. Considerando esses resultados, salienta-se a necessidade urgente de fiscalização e regulamentação oficial por parte das autoridades locais competentes, a fim de melhorar a qualidade higiênico-sanitária das hortaliças ofertadas à população e minimizar os índices de parasitoses em adultos e crianças (as mais afetadas).

Palavras-chave: Enteroparasitas. Agrião. Almeirão.

\begin{abstract}
The vegetable diseases transmitted represents a major public health problem, because it is estimated that millions of people worldwide are affected by parasites. This study aimed to quantify and identify the rate of contamination by helminth eggs on vegetables like watercress and endive marketed in four major supermarkets of Apucarana (PR). Were analyzed 128 sample of vegetables being: 64 of chicory and 64 of watercress, which were trimmer, weighed and washed in a tryptose lauryl sulfate $1 \%$ solution. There was a high rate of contamination in both varieties studied, with higher prevalence of chicory. Were found eggs of Ancylostoma sp., Ascaris sp. Diphilobotrium sp. Dipylidium sp. Hymenolepis sp. Taenia sp. and Enterobius sp. Considering these results, we stress the urgent need for official oversight and regulation by the competent local authorities in order to improve the hygienic quality of vegetables offered to the population, and minimize the rates of parasitic infections in adults and children (the most affected).
\end{abstract}

Keywords: Parasites. Watercress. Chicory.

${ }^{1}$ Aluna do Curso de Especialização em Biotecnologia - Universidade Estadual de Maringá (UEM), Maringá, (PR). E-mail: angelicafrias16@hotmail.com

${ }^{2}$ Doutoranda do Programa de Pós Graduação em Ciências da Saúde - Universidade Estadual de Maringá (UEM), Maringá, (PR). E-mail: joseanebalan@gmail.com

${ }^{3}$ Doutoranda do Programa de Pós Graduação em Ciência Ambiental - Universidade de São Paulo (USP), São Paulo (SP). E-mail: htozato@gmail.com 


\section{Introdução}

No Brasil, nos últimos anos o consumo de hortaliças in natura tem aumentado (FALAVIGNA et al., 2005) e, segundo Abreu (2008), esse fator está relacionado, principalmente, ao aumento populacional desordenado e à procura por alimentos mais saudáveis, com baixo custo e de fácil acesso.

Essa mudança também se vincula aos problemas de saúde pública do país, uma vez que essas hortaliças podem ser veículos de disseminação de cistos, ovos e larvas de enteroparasitas causadores de doenças (SOBRINHO; COELHO; OLIVEIRA, 1995; GUILHERME et al., 1999; MESQUITA et al., 1999; COELHO et al., 2001).

De acordo com Evangelista (2005), essa disseminação de agentes patogênicos de enfermidades entéricas acontece por meio de vegetais folhosos in natura consumidos na forma de saladas, principalmente em estabelecimentos de refeições coletivas como restaurantes (GERMANO; MARIA, 2001). Para proteger os consumidores, a Organização Mundial da Saúde (WORLD HEALTH ORGANIZATION, 1989) determina que as águas de irrigação podem apresentar 1 ovo/litro de nematóide e a legislação brasileira regulamentada pela Agência Nacional de Vigilância Sanitária (ANVISA) (Resolução no 12, de 1978) estabelece que as verduras devem apresentar, quanto às suas características microscópicas, ausência de sujidades, parasitas e larvas (BRASIL, 1978).

Entretanto, segundo Nolla e Cantos (2005), estima-se que milhões de pessoas de todo o mundo estejam acometidas por parasitoses e no Brasil a situação não é diferente. Seger et al. (2010) realizaram um estudo parasitológico em fezes de 121 adultos com idade superior a 30 anos, moradores da cidade de São Miguel do Oeste (SC) e observaram que, das amostras analisadas, 7,6\% apresentaram positividade para ovos de parasitos intestinais. Resultados semelhantes foram obtidos por Peres et al. (2009) em dois Centros de Saúde da cidade de Sorocaba, onde houve a presença de
Enterobius vermicularis, Ascaris lumbricoides, Giadia dudeonalis, Entamoeba coli e Endolimax nana. Na cidade de Maringá (PR), Guilherme et al. (1999) também detectaram que 26,4\% da população estudada apresentou uma ou mais espécies de parasitas, com maior prevalência de ancilostomídeos.

Essa elevada taxa de infecção relaciona-se também ao desconhecimento dos mecanismos de transmissão de enteroparasitoses (GIATTI; ROCHA; SANTOS, 2004). Guilherme et al. (2004) entrevistaram famílias de três vilas rurais no noroeste do Paraná, e observaram que 50\% dos entrevistados desconheciam formas de contaminação, quadro clínico e prevenção relacionados às parasitoses.

Sendo assim, o diagnóstico de enteroparasitas em hortaliças é de extrema importância para a saúde pública, pois fornece dados para vigilância sanitária e para a população sobre o real estado higiênico desses vegetais e permite um possível controle dos alimentos disponíveis ao consumidor (ALMEIDA FILHO, 2008).

O presente estudo teve por objetivo quantificar e identificar o índice de contaminação em hortaliças dos tipos agrião (Nasturtium officinale) e almeirão (Cichorium intybus L.) do município de Apucarana (PR), por ovos de helmintos.

\section{Material e Métodos}

A pesquisa foi realizada no período de maio de 2008 a setembro de 2009. Foram analisadas 128 amostras de hortaliças, sendo 64 de almeirão (C. intybus L.) e 64 de agrião ( $N$. officinale) de quatro supermercados do município de Apucarana (PR). As amostras foram coletadas e embaladas individualmente em sacos de polietileno, evitando assim possíveis contatos manuais e contaminações cruzadas. As mesmas foram armazenadas em caixa de isopor devidamente fechada e transportadas até o laboratório de parasitologia da Faculdade de Apucarana. 
Os maços de hortaliças foram desfolhados em meio estéril e $100 \mathrm{~g}$ de folhas de cada maço foram pesados em balança semi-analítica. As folhas foram submersas em béquer de vidro contendo $250 \mathrm{ml}$ de solução de Lauril Sulfato Triptose a $1 \%$ estéril, onde realizou-se o processo de lavagem com intuito de desprender as possíveis estruturas parasitárias.

As folhas lavadas foram desprezadas e a solução resultante da lavagem foi filtrada em gaze estéril depositada em um cálice de sedimentação, onde o líquido permaneceu neste processo durante 24 horas, segundo a técnica descrita por Hoffmann, Pons e Janer (1934).

Após o período de sedimentação, foi coletado 0,1 ml da sedimentação de cada amostra com auxílio de pipeta Pasteur, o qual foi colocado em lâmina de microscopia tamanho 25,4 x 76,2mm e corado por Lugol. As lâminas foram analisadas em microscópio óptico em aumentos de 10x e 40x. Adotou-se o método quantitativo para análise de ovos de helmintos.

\section{Resultados e Discussão}

Entre as 128 amostras analisadas, foi observado $50 \%$ de positividade, sendo 56,25\% nas amostras de almeirão e 43,75\% nas amostras de agrião. Em $27,77 \%$ das amostras de almeirão foi observado monoparasitismo (helminto), e em 72,23\% das amostras foi observado poliparasitismo (helmintos). Nas amostras de agrião, obteve-se $50 \%$ de monoparasitismo e $50 \%$ de poliparasitismo (Tabela 1).

Tabela 1 - Análises parasitológicas de duas variedades de hortaliças (almeirão e agrião) comercializadas em supermercados da cidade de Apucarana (PR), 2008-2009.

\begin{tabular}{lccccc}
\hline & $\mathbf{n}^{\circ} \mathbf{A . A}$. & $\mathbf{n}^{\circ} \mathbf{A . P}$. & $\mathbf{\% A . P .}$ & $\mathbf{\% M P}$ & \%PP \\
\hline Almeirão & 64 & 36 & 56,25 & 27,77 & 72,23 \\
Agrião & 64 & 28 & 43,75 & 50,00 & 50,00 \\
\hline TOTAL & 128 & 64 & 50,00 & 37,50 & 62,50 \\
\hline
\end{tabular}

$\mathrm{n}^{\circ} \mathrm{A} . \mathrm{A}=$ número de amostras analisadas; $\mathrm{N}^{\circ} \mathrm{A} . \mathrm{P} .=$ número de amostras positivas; $\% \mathrm{~A} . \mathrm{P}=$ porcentagem de amostras positivas; $\mathrm{MP}=$ monoparasitismo; $\mathrm{PP}=$ poliparasitismo.

Fonte: Autor.

Foi detectada a presença de 363 ovos de (2,20\%). No agrião havia Ascaris sp. (80,23\%), helmintos no almeirão, sendo a maior frequência Taenia sp. (6,60\%), Dipylidium sp. (5,50\%), de Ascaris sp. (2,20\%), seguido de Hymenolepis sp. Diphilobotrium sp. (3,29\%), Hymenolepis sp. (36,09\%), Dipylidium sp. (15,98\%), Diphilobotrium (3,29\%) e Enterobius sp.(1,09\%), totalizando 91 sp. (4,14\%), Taenia sp. (3,58\%) e Ancylostoma sp. ovos (Figura 1). 
Figura 1 - Helmintos encontrados em amostras de almeirão e agrião comercializadas no município de Apucarana (PR).

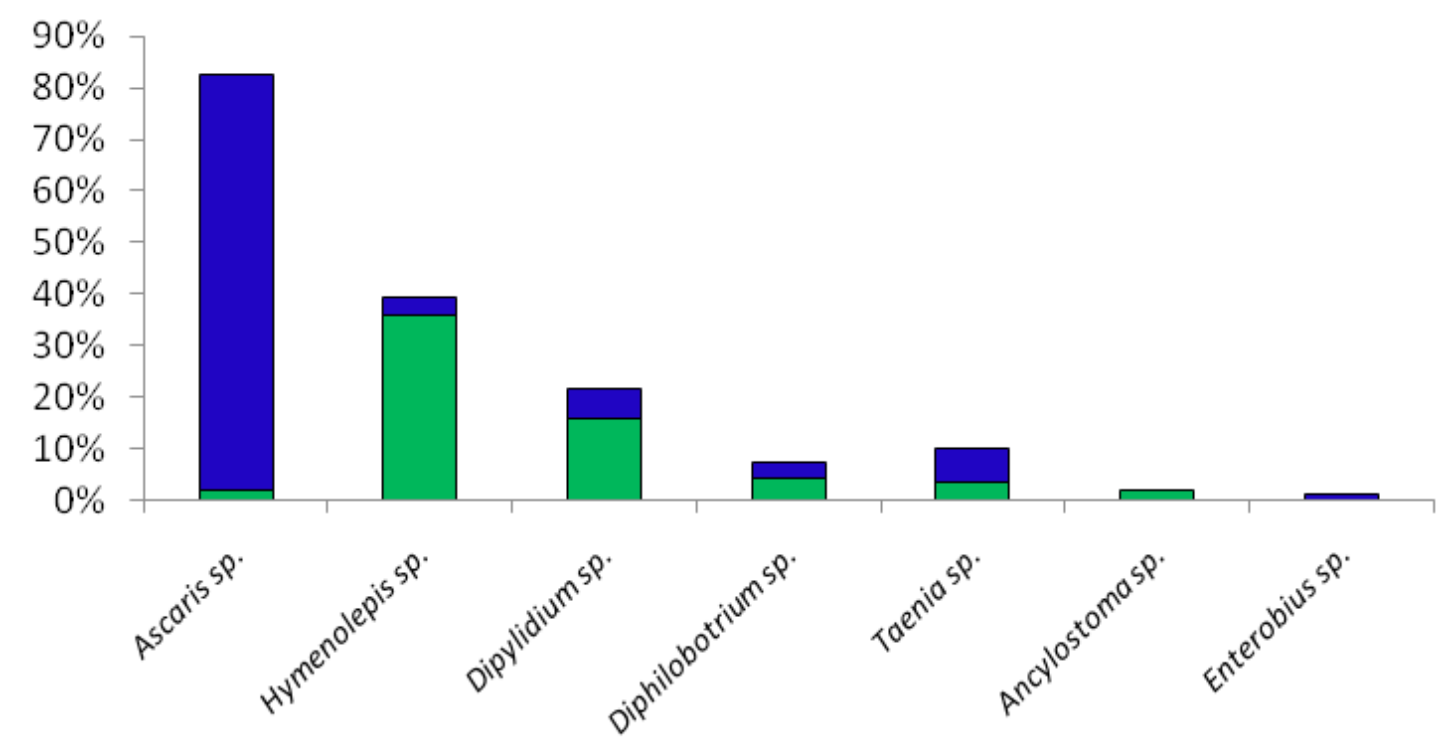

Almeirão Agrião

Fonte: Autor

Embora o índice de estruturas parasitárias tenha diferido entre as amostras de almeirão e agrião, Ascaris sp. apresentou-se como o helminto de maior frequência nas duas hortaliças. Resultados semelhantes foram descritos por Freitas et al. (2004), que identificaram a maior frequência desse patógeno em hortaliças comercializadas na cidade de Campo Mourão (PR).

A elevada frequência de Ascaris sp. pode estar relacionada à morfologia de sua casca, a qual é constituída de mucopolissacarídeo que promove sua adesão às folhagens dos vegetais, como também à sua resistência a altas e baixas temperaturas no solo, o que pode facilitar sua sobrevivência e disseminação (COELHO et al., 2001). O Ascaris sp. é considerado helminto de maior preocupação em saúde pública, pois estima-se que esteja presente em 1/4 da população mundial (CROMPTON, 1999) e pode causar quadros sumamente graves e fatais (O’LORCAIN; HOLLAND, 2000).

Pode-se observar, ainda no presente estudo, a ocorrência de Hymenolepis sp. com elevada frequência nas amostras de almeirão, sugerindo que sua presença nas hortaliças pode ter acontecido pelo uso de água ou adubo contaminados por excretas de humanos ou animais portadores deste patógeno.

Em relação à Diphilobotrium sp., não houve grande variação na frequência entre as duas variedades de hortaliças analisadas. Este mesmo organismo foi detectado em amostras de agriões coletadas em feiras livres da cidade Florianópolis (SC) (CANTOS et al., 2004).

Nas analises no município de Apucarana (PR), foi detectada a presença de ovos de Taenia sp. nas duas variedades de vegetais. O ovo de T. solium quando ingerido pode causar cisticercose humana, com quadros de convulsões e hipertensão intracraniana entre outros (SILVA-VERGARA et al., 1998). O porco e o boi são hospedeiros obrigatorios de $\mathrm{T}$. solium e T. saginata, respectivamente, diante disso, possivelmente a contaminação do vegetal pode ter ocorrido com uso de excretas de bovinos ou suínos (CORTÊS, 2000). 
Takayanagui et al. (2007) encontraram ancilostomídeos em almeirões de hortas de Ribeirão Preto (SP) e Cantos et al. (2004) registraram a presença de larvas e ovos de ancilostomídeos em agriões. No presente estudo foram detectados ovos de Ancylostoma sp. apenas no almeirão e com baixa frequência. $\mathrm{O}$ percentual de ovos deste gênero foi semelhante ao observado por Takizawa, Falavigna e Gomes (2009) em fezes de manipuladores de alimentos da cidade de Cascavel (PR).

Em um estudo realizado por Rocha, Mendes e Barboza (2008) em alfaces orgânicas e convencionais comercializadas em supermercados e feiras livres da cidade Recife (PE), foi observada baixa frequência de ovos de Ancylostoma sp. em hortaliças das feiras e ausência destes nas amostras dos supermercados.

Enterobius sp. foi o helminto de menor incidência encontrado no agrião. No Brasil, vários autores realizaram estudos parasitológicos em fezes humanas para diagnosticar incidência de enteroparasitas, sendo que os resultados demonstraram ausência ou baixa frequência de Enterobius sp. (CARDOSO et al., 2010; MACEDO et al., 2010; MIRANDA; DATOLLI; LIMA, 2010).

\section{Considerações}

Considerando o Plano Nacional de Vigilância e Controle das Enteroparasitoses da Secretaria de Vigilância e Saúde do Ministério da Saúde (BRASIL, 2005), os organismos encontrados no presente estudo possivelmente são Ascaris lumbricoides, Hymeonolepis nana e/ou $H$. diminuta, Enterobius vermiculares, Taenia saginata e/ou T. solium. Essas espécies são de grande relevância em saúde pública, pois possuem etiologia patogênica (SANTANA et al., 2006) na qual os indivíduos mais afetados constituem-se grupos pediátricos em idade escolar, populações de baixo poder aquisitivo e regiões onde há falta de saneamento básico e água tratada (MACHADO et al., 1999).

Embora a Resolução CNNPA n ${ }^{\text {12, de }} 1978$ da
Agência Nacional de Vigilância Sanitária (ANVISA) determine que as características microscópicas de verduras sejam ausência de sujidades, parasitas e larvas, o presente estudo confirmou a elevada incidência de enteroparasitas em agriões e almeirões comercializados em Apucarana (PR). Isso significa que comerciantes do município encontram-se realizando atividades em desacordo com a legislação vigente.

Essa contaminação de hortaliças pode estar associada aos fatores de disseminação de formas parasitárias como àquelas citadas por Mesquita et al. (1999) e Coelho et al. (2001), como presença de formas parasitárias no solo, na água de irrigação, na água de lavagem dos vegetais e mesmo no seu processo de manipulação na colheita, reposição e venda.

O presente estudo ressalta a necessidade urgente de fiscalização e regulamentação oficial por parte dos órgãos locais competentes, a fim de melhorar a qualidade higiênico-sanitária das hortaliças ofertadas à população e minimizar os índices de parasitoses na população em geral.

\section{Agradecimentos}

À Faculdade de Apucarana pela disponibilização de laboratório. Às alunas Karine Silvestre, Amanda Santos Burin, Nayara Faila e Francielle Lima Vidotto pelo auxílio nas análises parasitológicas.

\section{Referências}

ABREU, I. M. O. Produtividade e qualidade microbiológica de alface sob diferentes fontes de adubos orgânicos. 2008. Dissertação (Mestrado em Ciências Agrárias) - Universidade de Brasília, Brasília.

ALMEIDA FILHO, P. C. Avaliação das condições ambientais e higiênico-sanitarias na produção de hortaliças folhosas no núcleo hortícula suburbano de Vargem Bonita, Distrito Federal. 2008. Dissertação (Mestrado em Planejamento e Gestão Ambiental) Universidade Católica de Brasília, Brasília. 
BRASIL. Comissão Nacional de Normas e Padrões para Alimentos. Agencia Nacional da Vigilância Sanitária. CNNPA/ANVISA. Normas técnicas especiais. n. 12, São Paulo, 1978.

Ministério da Saúde. Plano nacional de vigilância e controle das Enteroparasitoses. Secretaria de Vigilância e Saúde, 2005. Disponível em: <http://portal. saude.gov.br/portal/arquivos/pdf/Enteroparasitoses Pano_nacional_\%2006\%2007\%202005.pdf.>. Acesso em: 9 set. 2011.

CANTOS, G. A.; SOARES, B.; MALISKA, C.; GLICK, D. Estruturas parasitárias encontradas em hortaliças comercializadas em Florianópolis, Santa Catarina. NewsLab, São Paulo, v. 66, p. 154-166, 2004.

CARDOSO, F. D. P.; ARAÚJO, B. M.; BATISTA, H. L.; GALVÃO, W. G. Prevalência de enteroparasitoses em escolares de 06 a 14 anos no município de AragauínaTocantins. Revista Eletrônica de Farmácia, Goiânia, v. 7, n. 1, p. 54-64, 2010.

COELHO, L. M. P. S.; OLIVEIRA, S. M.; MILMAN, M. H. S. A.; KARASAWA, K. A.; SANTOS, R. P. Detecção de formas transmissíveis de enteroparasitas na água e nas hortaliças consumidas em comunidades escolares de Sorocaba, São Paulo, Brasil. Revista da Sociedade Brasileira de Medicina Tropical, Rio de Janeiro, v. 34, n. 5, p. 479-482, 2001.

CÔRTES, J. A. Complexo teníase humana: cisticercose bovina e suína. Revista Educação Continuada, São Paulo, v. 3, n. 2, p. 61-71, 2000.

CROMPTON, D. W. T. How much human helminthiasis is there in the world? Journal of Parasitology, Lawrence, v. 85 , p. $397-493,1999$.

EVANGELISTA, J. Alimentos: um estudo abrangente. São Paulo: Atheneu, 2005.

FALAVIGNA, L. M.; FREITAS, C. B. R.; MELO, G. C.; NISHI,L.; ARAÚJO, S. M.; GUILHERME,A. L. F. Qualidade de hortaliças comercializadas no noroeste do Paraná, Brasil. Parasitología latinoamericana, Santiago, v. 60, p. 144-149, 2005.

FREITAS, A. A.; KWIATKOWSKI, A.; NUNES, S. C.; SIMONELli, S. M.; SANGIONI, L. A. Avaliação parasitológica de alfaces (Lactuca sativa) comercializadas em feiras livres e supermercados do município de Campo Mourão, Estado do Paraná. Acta Scientiarum: Biological Sciences, Maringá, v. 26, n. 4, p. 381-384, 2004.

GERMANO, P. M. L.; MARIA, I. S. G. Higiene e vigilância sanitárias de alimentos. São Paulo: Varela, 2001.

GIATTI, L. L.; ROCHA, A. A.; SANTOS, F. A.;
BITENCOURT, S. C.; PIERONI, S. R. M. Condições de saneamento básico em Iporanga, Estado de São Paulo. Revista Saúde Pública, São Paulo, v. 38, n. 4, p. 571-577, 2004.

GUILHERME, A. L. F.; ARAÚJO, S. M.; FALAVIGNA, D. L. M.; PUPUlIM, A. R. T.; DIAS, M. L. G. G.; OLIVEIRA, H. S.; MAROCO, E.; FUKUSHIGUE, Y. Prevalência de enteroparasitas em horticultores e hortaliças da feira do produtor de Maringá, Paraná. Revista da Sociedade Brasileira de Medicina Tropical, Rido de Janeiro, v. 32, n. 4, p. 405-411, 1999.

GUILHERME, A. L. F.; ARAÚJO, S. M.; PUPULIM, A. R. T.; LIMA JUNIOR, J. E.; FALAVIGNA, D. L. M. Parasitas intestinais e comensais em indivíduos de três vilas rurais do estado do Paraná, Brasil. Acta Scientiaurum: Health Sciences, Maringá, v. 26, n. 2, p. 331-336, 2004.

HOFFMANN, W. A.; PONS, J. A.; JANER, J. L. The sedimentation concentration method in schistosomiasis mansoni. Puerto Rico Journal of Public Health and Tropical Medicine, New York, v. 9, p. 283-291, 1934.

MACEDO, H. W.; GONÇALVES, A. M. H.; ALMEIDA, C. A.; DIAS, L. V. B.; MUNIZ, M. F. Infecção por Blastocystis hominis e Entamoeba histolytica/Entamoeba díspar em pacientes atendidos em um hospital localizado em Niterói, Rio de Janeiro. Revista de Patologia Tropical, Goiânia, v. 39, n. 1, p. 56-62, 2010.

MACHADO, R. C.; MARCARI, E. L.; CRISTANTE, S. F. V.; CARARETO, C. M. A. Giardíase e helmintíase em crianças de creches e escolares de $1^{\circ} \mathrm{e} 2^{\circ}$ graus (públicas e privadas) da cidade de Mirassol (SP, BRASIL). Revista da Sociedade Brasileira de Medicina Tropical, Rio de Janeiro, v. 32, n. 6, p. 697-704, 1999.

MESQUITA, V. C. C.; SERRA, C. M. B.; BASTOS, O. M. P.; UCHÔA, C. M. A. Contaminação por enteroparasitas em hortaliças comercializadas nas cidades de Niterói e Rio de Janeiro, Brasil. Revista da Sociedade Brasileira de Medicina Tropical, Rio de Janeiro, v. 34, n. 4, p. 189194, 1999.

MIRANDA, G. C.; DATOLLI, V. C.; LIMA, A. D. Enteroparasitos e condições socioeconômicas e sanitárias em uma comunidade quilombola do semiárido baiano. Revista de Patologia Tropical, Goiânia, v. 39 n. 1, p. 4855, 2010.

NOLlA, A. C.; CANTOS, G. A. Relação entre a ocorrência de enteroparasitos em manipuladores de alimentos e aspectos epidemiológicos em Florianópolis, Santa Catarina, Brasil. Revista Caderno de Saúde Pública, Rio de Janeiro, v. 21, n. 2, p. 641-645, 2005. 
O'LORCAIN, P.; HOLLAND, C. V. The public health importance of Ascaris lumbricoides. Parasitology, Cambridge, v. 121, p. 51-71, 2000.

PERES, E. A.; IVANA, V. C.; BARELLA, W.; CORNÉLIA, M. Ocorrencia de parasitos intestinais e intervencao educativa em uma escola estadual na cidade de Sorocaba. Revista Eletrônica de Biologia, São Paulo, v. 2, n. 4, p. 26-37, 2009.

ROCHA, A.; MENDES, R. A.; BARBOZA, C. S. Strongyloides spp e outros parasitos encontrados em alfaces (Lactuta sativa) comercializados na cidade de Recife, PE. Revista de Patologia Tropical, Goiânia, v. 37, n. 2, p. 151-160, 2008.

SANTANA, L. R. R.; CARVALHO, R. D. S.; LEITE, C. C. L.; ALCÂNTRA, L. M.; OLIVEIRA, T.W. S.; RODRIGUES, B. M. Qualidade física, microbiológica e parasitológica de alfaces (Lactuta sativa) de diferentes sistemas de cultivo. Ciência e Tecnologia de Alimentos, Campinas, v. 26, n. 2, p. 246-269, 2006.

SEGER, J.; SOUZA, W. M.; MARANGONI, J. C. F.; MASCHIO, V. J.; CHIELLI, E. O. Prevalência de parasitas intestinais na população do bairro Salete, município de São Miguel do Oeste, SC. Unoesc \& Ciência: $A C B S$, Joaçaba, v. 1, n. 1, p. 53-56, 2010.

SILVA-VERGARA, M. L.; PRATA, A.; VELLOSO, H.; NETTO, S.; VIEIRA, C. O.; CASTRO, J. H.; MICHELETTI, L. G.; OTAÑO, A. S.; JÚNIOR, J. F. Risk factors associated with taeniasis-cysticercosis in Lagamar, Minas Gerais State, Brazil. Revista da Sociedade Brasileira de Medicina Tropical, Rio de Janeiro, v. 31, n. 1, p. 65-71, 1998.

SOBRINHO, T. A.; COELHO, L. M.; OLIVEIRA, S. M. Estudo da freqüência de ovos de helmintos intestinais em sanitários de uso público de Sorocaba, SP. Revista da Sociedade Brasileira de Medicina Tropical, Rio de Janeiro, v. 28, p. 33-37, 1995.

TAKAYANAGUI, O. M.; CAPUANO, D. M.; OLIVEIRA, C. D.; BERGAMINI, A. M.; OKINO, M. H. T.; SILVA, A. A. C. C. E.; OLIVEIRA, M. A.; RIBEIRO, E. G. A.; TAKAYANAGUI, A. M. M. Avaliação da contaminação de hortas produtoras de verduras após a implantação do sistema de fiscalização em Ribeirão Preto, SP. Revista da Sociedade Brasileira de Medicina Tropical, Rio de Janeiro, v. 40, p. 239-241, 2007.

TAKIZAWA, M. G. M. H.; FALAVIGNA, D. L. M.; GOMES, M. L. Enteroparasitas em materiais fecal e subungueal de manipuladores de alimentos, Estado do Paraná, Brasil. Acta Scientiarum: Health Sciences, Maringá, v. 31, n. 2, p. 89-94, 2009.

WORLD HEALTH ORGANIZATION - WHO. Health guidelines for the use of wastewater in agriculture and aquaculture: technical report series. Geneva: World Health and Organization, 1989.

Recebido em 13 de setembro de 2011

Aceito em 30 de setembro de 2011 
